\title{
El cambio en el razonamiento bayesiano de estudiantes universitarios durante un semestre académico' ${ }^{1}$
}

\author{
The change in the Bayesian reasoning university \\ students during an academic semester
}

\section{A mudança nos Bayesian estudantes universitários de raciocínio durante um semestre acadêmico}

Recibido: mayo de 2013

Aceptado: agosto de 2013
Cindy Nathalia Morgado Hernández ${ }^{2}$

Gabriel Yáñez $\mathrm{Canal}^{3}$

\section{Resumen}

En este trabajo se presentan los avances de una investigación que estudia el cambio en el razonamiento bayesiano en estudiantes universitarios que toman un primer curso de estadística. Se desarrolla un estudio longitudinal con 102 estudiantes. Se diseñan tres pruebas para evaluar el razonamiento, las cuales se aplican en momentos diferentes durante el semestre. Un análisis descriptivo de los resultados obtenidos muestra que la enseñanza de los conceptos de probabilidad condicional y teorema de Bayes tuvieron un efecto en su razonamiento, esta influencia mostró crecimiento, pero con el tiempo su razonamiento decae, lo que se evidenció en los resultados bajos obtenidos al final del semestre, los cuales fueron aún más bajos que los resultados al inicio del semestre.

Palabras clave: Matemáticas escolares; estadística; inferencia; razonamiento bayesiano; evaluación; datos longitudinales.

\begin{abstract}
In this paper we present progress of an investigation that studies the change in the Bayesian reasoning in college students taking a first course in statistics. It develops a longitudinal study with 102 students. Three tests are designed to assess reasoning, which apply at different times during the semester. A descriptive analysis of the results shows that the teaching of the concepts of conditional probability and Bayes theorem had an effect on his reasoning, this influence was growing, but eventually decays reasoning, which was evidenced by the low results obtained at the end of the semester, which were even lower than the results at the beginning of the semester.
\end{abstract}

Keywords: Mathematical school; statecraft; inference, Bayesian reasoning; evaluación; datos longitudinal.

Articulo de Investigación

Universidad Industrial de Santander, Colombia. Contacto: cinathalia@gmail.com

Universidad Industrial de Santander, Colombia. Contacto: gyanez@uis.edu.co 


\section{Resumo}

Neste artigo, apresentamos o progresso de uma investigação que estuda a mudança no raciocínio Bayesian em estudantes universitários que tomam um primeiro curso em estatística. Desenvolve-se um estudo longitudinal com 102 alunos. Três testes foram projetados para avaliar o raciocínio, que se aplicam em diferentes momentos durante o semestre. A análise descritiva dos resultados mostra que o ensino dos conceitos de probabilidade condicional e teorema de Bayes teve um efeito sobre seu raciocínio, essa influência foi crescendo, mas o raciocínio eventualmente decai, o que foi evidenciado pelos baixos resultados obtidos no final do semestre, que foram ainda menores do que os resultados no início do semestre.

Palavras-chave: Matemáticas ESCOLARES; Estadística; inferencia; razonamiento bayesiano; evaluación; datos longitudinales.

\section{Introducción}

El razonamiento bayesiano hace referencia al cálculo de probabilidades condicionales inversas mediante el teorema de Bayes, el cual permite, a través de las probabilidades iniciales y de los datos obtenidos experimentalmente, obtener las probabilidades finales. La presentación estándar de este teorema se centra sobre la probabilidad condicional de dado los datos.

Donde es la probabilidad de, y es la probabilidad condicional de dado el evento .

La probabilidad condicional y, en particular, el teorema de Bayes, han sido objeto de muchas investigaciones en didáctica de la probabilidad que dan cuenta de algunas de las dificultades que presentan los estudiantes en la comprensión de estos temas. Ciertas investigaciones mostraron que los alumnos en su mayor parte no tienen en cuenta las probabilidades a priori en el cálculo de la probabilidad inversa (Kahneman y Tversky, 1972). Los estudiantes confunden el evento condicionante con el condicionado dando lugar a la falacia de la condicional transpuesta, es decir confunden con la (Falk, 1986). Adicionalmente se interpreta la probabilidad condicional como una relación temporal, donde el suceso condicionante B siempre precede al suceso A (Gras y Totohasina, 1995) y las mayores fuentes de error es la confusión entre las probabilidades condicionales y las probabilidades conjuntas (Pollatsek y cols., 1987). De otro lado se conoce que la aplicación del teorema de Bayes es más sencilla cuando la información se da en frecuencias absolutas, en lugar de usar probabilidades, porcentajes o frecuencias relativas. (Gigerenzer y Hoffrage, 1995) y los errores en la solución de problemas se producen en diferentes pasos del proceso de resolución, comenzando por la identificación correcta de los sucesos y sus probabilidades, y la correcta partición y subpartición del espacio muestral (Díaz y de la Fuente, 2006).

\section{Presentación del problema}

Las investigaciones sobre el razonamiento bayesiano en el campo de la educación estadística se resumen en las dificultades que presentan los estudiantes al enfrentarse a problemas de probabilidad condicional, sobre las intuiciones y concepciones que se forman sobre los conceptos asociados y sobre las representaciones utilizadas en la resolución de problemas. Recientemente el interés de investigación se ha dirigido a conocer el efecto que tienen los formatos de presentación de la información sobre el éxito en la resolución de problemas (Lonjedo y Huerta, 2007).

Por otra parte, las investigaciones sobre el razonamiento bayesiano, así como de gran parte de la literatura en investigación en matemática educativa, han sido principalmente de corte transversal. De hecho, en la literatura revisada no se encontró ningún estudio longitudinal acerca del razonamiento bayesiano. Motivados por esta situación diseñamos 
una investigación que permitiera responder la siguiente pregunta: ¿Cómo cambia el razonamiento bayesiano en estudiantes universitarios que toman un curso de probabilidad y estadística?

\section{Metodología}

Con el fin de dar respuesta a la pregunta de investigación, se desarrolla un estudio longitudinal con estudiantes universitarios que toman por primera vez un curso de probabilidad y estadística. Se plantea el diseño de 3 pruebas para evaluar el razonamiento bayesiano, las cuales fueron aplicadas en diferentes momentos en el semestre. Con los resultados obtenidos en las diferentes pruebas se obtendrán puntajes, los cuales, se analizarán para conocer el cambio en el razonamiento bayesiano de los estudiantes.

Diseño de los instrumentos. El procedimiento consistió en elaborar un listado con el contenido implícito en la fórmula de Bayes como es: la probabilidad condicional, la probabilidad marginal, el teorema de probabilidad total y la regla del producto.

Para la primera prueba se tomaron 14 ítems del cuestionario diseñado por Díaz (2007) teniendo en cuenta la descomposición del constructo. La aplicación de esta prueba se realizó en la primera semana de clase en todos los cursos.

El segundo y tercer instrumento se diseñaron con base en el primer instrumento. Los problemas se seleccionaron de libros de texto y páginas web, los cuales se modificaron con el fin de evaluar el concepto deseado. La segunda prueba se aplicó en la doceava semana del semestre y la tercera prueba en la última semana del semestre en todos los cursos. Es importante destacar que la duración de los cursos fue de 16 semanas.

Muestra. Los sujetos son 102 estudiantes de dos universidades diferentes, que por primera vez toman una asignatura donde el contenido es probabilidad y estadística básica. Es de resaltar, que la asignatura en cada una de las universidades recibe nombres diferentes (Bioestadística I y Estadística para Ingenieros).
De Estadística para Ingenieros, 51 son estudiantes que están conformados por dos grupos, 32 estudiantes del grupo A1 y 19 estudiantes el grupo A2. En estos grupos un mismo profesor les imparte la asignatura. De Bioestadística I, 51 estudiantes que están conformados por dos grupos, 38 estudiantes del grupo B1 y 13 estudiantes del grupo B2. En estos grupos un mismo profesor les imparte la asignatura.

\section{Resultados y Discusión}

Se realiza el análisis descriptivo de las respuestas correctas dadas por los estudiantes a cada prueba, asignando una calificación entre 0 y 14 en cada prueba.

La Figura 1 muestra el factor puntaje de respuestas correctas de los estudiantes para los tres momentos (en el eje vertical se muestra la media de las respuestas) y los niveles grupos (A1 y A2) del curso de Estadística para Ingenieros. La Figura 2 muestra el factor puntaje de respuestas correctas de los estudiantes para los tres momentos y los niveles grupos (B1 y B2) del curso de Bioestadística I.

Figura 1. Diagrama de interacción de la media de las respuestas correctas versus el tiempo para cada grupo del curso de Estadística para Ingenieros.

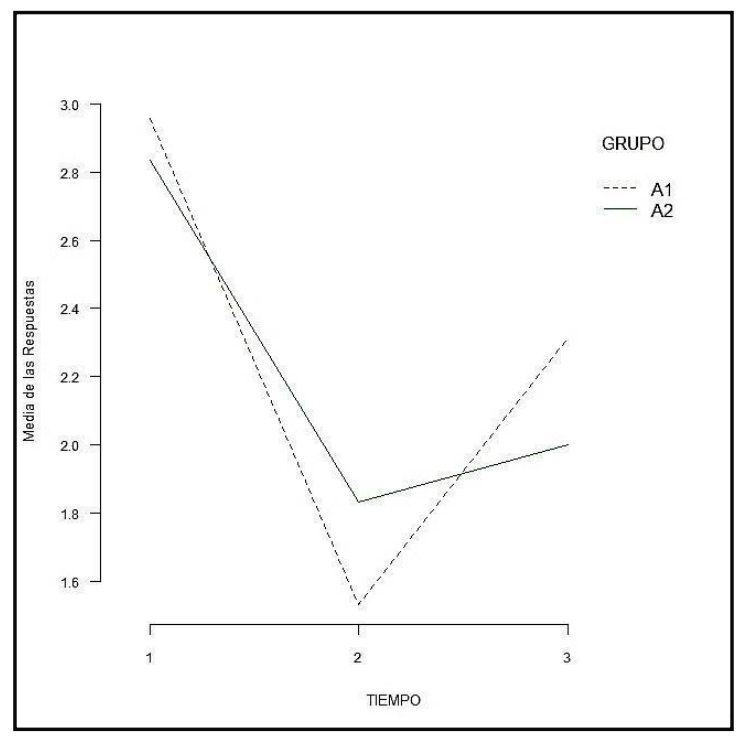

Fuente: Elaboración propia

En la Figura 1 se observan en el primer momento que la media de las respuestas es mayor para el grupo A1, en el segundo momento se observa una 
disminución en la media de las respuestas para los dos grupos, situación bastante anómala ya que se esperaba que la media de las repuestas aumentara. Sin embargo, la media para el curso A2 es mayor en el segundo y tercer momento. Se resalta el hecho que en el tercer momento la media de las respuestas fue más bajo que las media de las respuestas en el primer momento, lo que evidencia que su razonamiento bayesiano no crece, incluso cuando ya han recibido formación formal, sino que se ve afectado por los conceptos que se les imparte durante el curso, es decir, no logran incorporar a sus intuiciones previas los conceptos que ven a través del curso. Además, ambos grupos tienen un comportamiento similar en los tres momentos.

Figura 2. Diagrama de interacción de la media de las respuestas correctas versus el tiempo para cada grupo del curso de Bioestadística I.

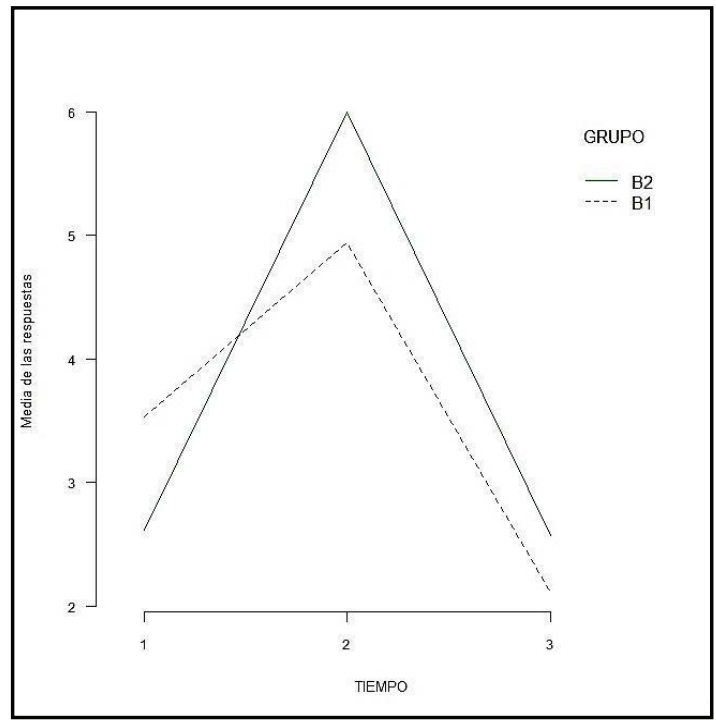

Fuente: Elaboración propia

En la Figura 2 se observa que la media de los puntajes del grupo B1 fue mayor, y en el segundo momento los puntajes de los dos cursos tuvieron un fuerte aumento, donde la media de las notas del grupo B2 fueron mayores a las del grupo B1 y disminuyeron en el tercer momento, donde se muestra que el grupo B1 obtuvo un puntaje final menor a del grupo B2. A pesar que en el tercer momento la media de las respuestas fue bajo, este puntaje fue cercano a media de las respuestas en el primer momento para el grupo B2. Por el contrario para el grupo B1 en el tercer momento la media de las respuestas fue más bajo que las media de las respuestas en el primer momento, lo que evidencia que su razonamiento bayesiano no crece, incluso cuando ya han recibido formación formal, sino que se ve afectado por los conceptos que se les imparte durante el curso.

\section{Referencias}

Díaz, C. (2007). Viabilidad de la enseñanza de la inferencia bayesiana en el análisis de datos en psicología. Tesis doctoral. Universidad de Granada

Díaz, C. y de la Fuente, I. (2006). Dificultades en la resolución de problemas que involucran el Teorema de Bayes. Un estudio exploratorio en estudiantes de psicología. Educación Matemática, 18 (2), 75-94.

Falk, R. (1986). Conditional probabilities: insights and difficulties. En R. Davidson y J. Swift (Eds.), Proceedings of the Second International Conference on Teaching Statistics. University of Victoria. 292 - 297.

Gras, R. y Totohasina, A. (1995). Chronologie et causalité, conceptions sources d'obstacles épistémologiques à la notion de probabilité conditionnelle. Recherches en Didactique des Mathématiques. 15 (1), 49 - 95.

Gigerenzer, G., y Hoffrage, U. (1995). How to improve Bayesian reasoning without instruction: Frequency formats (pp. 129-161). Psychological Review, 102, $684-704$.

Kahneman y Tversky (1972) Subjective probability: A judgment of representativeness. Cognitive Psychology 3:430-454

Lonjedo, Ma A. y Huerta, M. P. (2007). Análisis del comportamiento de los estudiantes en la resolución de problemas isomorfos de probabilidad condicional. En Camacho, Flores y Bolea (Eds) Investigación en educación matemática XI, 273-282

Pollatsek, A., Well, A. D., Konold, C. y Hardiman, P. (1987). Understanding conditional probabilities. Organization, Behavior and Human Decision Processes, 40, 255 - 269. 